\title{
Dendrimer-paclitaxel complexes for efficient treatment in ovarian cancer: study on OVCAR-3 and HEK293t cells
}

\author{
Hua Yao ${ }^{1}$ and Jinqi Ma ${ }^{\varpi}$ \\ 'Department of Gynecology and Obstetrics, The second People's Hospital of Nantong, Nantong, Jiangsu 226002, China; ${ }^{2}$ Department of Obstet- \\ rics, Wuxi People's Hospital, Wuxi, Jiangsu 214023, China
}

The present paper investigates the enhancement of the therapeutic effect of Paclitaxel (a potent anticancer drug) by increasing its cellular uptake in the cancerous cells with subsequent reduction in its cytotoxic effects. To fulfill these goals the Paclitaxel (PTX)-Biotinylated PAMAM dendrimer complexes were prepared using biotinylation method. The primary parameter of Biotinylated PAMAM with a terminal $\mathrm{HN}_{2}$ group - the degree of biotinylation - was evaluated using HABA assay. The basic integrity of the complex was studied using DSC. The Drug Loading (DL) and Drug Release (DR) parameters of Biotinylated PAMAM dendrimer-PTX complexes were also examined. Cellular uptake study was performed in OVCAR-3 and HEK293T cells using fluorescence technique. The statistical analysis was also performed to support the experimental data. The results obtained from HABA assay showed the complete biotinylation of PAMAM dendrimer. DSC study confirmed the integrity of the complex as compared with pure drug, biotinylated complex and their physical mixture. Batch 9 showed the highest DL (12.09\%) and DR (70\%) for $72 \mathrm{~h}$ as compared to different concentrations of drug and biotinylated complex. The OVCAR-3 (cancerous) cells were characterized by more intensive cellular uptake of the complexes than HEK293T (normal) cells. The obtained experimental results were supported by the statistical data. The results obtained from both experimental and statistical evaluation confirmed that the biotinylated PAMAM $\mathrm{NH}_{2}$ dendrimer-PTX complex not only displays increased cellular uptake but has also enhanced release up to $72 \mathrm{~h}$ with the reduction in cytotoxicity.

Key words: Paclitaxel, Biotinylated PAMAM $\mathrm{NH}_{2}$ complex, ovarian cancer, cellular uptake, cytotoxicity.

Received: 29 September, 2017; revised: 20 October, 2017; accepted: 21 October, 2017; available on-line: 18 June, 2018

e-mail: jinqima138@gmail.com

Abbreviations:

\section{INTRODUCTION:}

Ovarian cancer is one of the major cause of cancer death in women (Razi et al., 2016). Risk of ovarian cancer in a lifetime is approximately $1.5 \%$ making it the second most common gynecological malignancy (Harley et al., 2008). The ovarian cancer tend to be comparatively aggressive with no proven early detection tests, most cases remain undiagnosed till advanced stages i.e. III or IV. Initially, the symptoms may be discrete or not recognizable but become distinguishable with the progression of the disease (Geggel \& Writer, 2014). Some of them may include abdominal pain, discomfort, back pain, ir- regular menstruation or postmenopausal vaginal bleeding, and possible urinary symptoms like frequent or urgent urination (Bankhead et al., 2005). The chances of ovarian cancer are increased in women who have ovulated more over their lifetime or who begin to ovulate at a younger age or reach menopause at an older age. Some other factors may include hormone therapy, fertility medications, and obesity (Moley \& Colditz, 2016; Zarchi et al., 2013). Women with a mutation in genes encoding $B R C A 1$ and $B R C A 2$ proteins have about $50 \%$ of chance of developing the disease (Petrucelli et al., 2016). On the other hand, hormonal birth control and breastfeeding help to reduce the incidence of ovarian cancer (Ferris et al., 2014). Removal of ovaries is one of the preventive measures for women in high-risk group (Lifford et al., 2012). The primary diagnosis starts with physical examination, blood test, transvaginal ultrasound imaging, and a test for cancer cells in the abdominal fluid. This helps to determine if the tumor is malignant or benign (Cragun, 2011). The confirmation of disease is made through a tissue biopsy. The treatment is usually a combination of 3 therapies i.e. surgery, radiation therapy and chemotherapy (Yap et al, 2009). Results depend on the extent and subtype of the disease. Surgery is performed to obtain tissue sample while radiation therapy is not effective for advanced stages due to the incapability of the vital organs to withstand it. Chemotherapy is considered a gold standard of care and generally used after the surgery to treat any residual disease (Rosen et al., 2014). Even after successful completion of the therapy, some individuals may experience the reoccurrence of the disease within two years. The resulting tumors frequently become tolerant to chemotherapy and extend into the peritoneal cavity. For these, the high dose regimen treatment is suggested (Nagel et al., 2012).

The major hurdle behind the treatment is lack of selectivity and multi-drug resistance resulting in a suboptimal concentration of drug in tissues (Wu et al., 2014). Therefore, a steady-state infusion of the particular chemotherapeutic agent is required (McDermott et al., 2014). Targeted delivery of anti-tumor agent with the aid of cell-specific ligand can prove beneficial for these resistant tumors. One of such useful ligand is biotin. Rapidlygrowing cancer cells can digest large amounts of molecules such as vitamins including biotin and vitamin B12. The biotin was found in higher amount in some cancer cells as compared to normal cells (Takechi et al., 2008). This targeted delivery can be performed with a chemotherapeutic agent like Paclitaxel (PTX). Biotin has recently emerged as targeting ligand which has the ability to increase the specificity of polymers for the cancerous cells (Yang et al., 2009). Various tumor cells including ovarian, colorectal, etc., showed increased biotin receptors up- 
take. It was stated that biotin-polymer conjugates can be internalized by endocytosis, due to the availability of free carboxyl group on the biotin molecule. The biotin receptors are also found to be co-expressed with those receptors involved in the folate (vitamin B12) uptake (Russel-Jones et al., 2004; Rao et al., 1997). Targeting of the drugs using biotin can be useful thanks to utilizing nanocarriers such as PAMAM dendrimers. The surface of the dendrimers has multiple functional groups which allow interaction of a large number of drug molecules per dendrimer, therefore increasing the drug loading and stability (Yang et al., 2009). Because of their very small size, strong electrostatic interactions take place between the charged surface groups and the cell membrane, which results in better loading capacity. Therefore, PAMAM dendrimers can turn out superior in comparison to the other polymeric nanoparticulate systems. The leaky tumor tissues provide enhanced permeability and retention (EPR) effect to the dendrimers. Thus, complexation of a drug with biotinylated PAMAM dendrimers could be an attractive way to improve its cellular uptake by the tumor cells (Kesharwani \& Iyer, 2015). The increase in cellular uptake of the drug can not only enhance its efficacy but also facilitate overcoming the drug resistance. Because of the positive surface charge, the observed cellular uptake of PAMAM G4 $\mathrm{NH}_{2}$ dendrimers was higher than of PAMAM G3.5 COOH dendrimers (Marek et al., 1997).

PTX is the first line chemotherapeutic agent which acts by disrupting normal microtubule breakdown during the cell division (Markman \& Mekhail, 2002). The major problem with the drug is its poor solubility in water (Narvekar et al., 2014). Thus, it is currently used as Taxol (a mixture of cremophor and absolute ethanol), but due to a high proportion of cremophor along with nonspecific bio-distribution (both in normal and tumor cells), it showed some serious side effects like hypersensitivity and neuro-sensitivity. Moreover, the resistance to PTX is observed in more than $70 \%$ of the patients. Therefore, it is worthy to develop alternate delivery systems for PTX which would increase its availability and maximize the therapeutic value (Anon, 1993).

\section{MATERIALS AND METHODS:}

Paclitaxel was obtained as a free sample from Shouguang Fukang Pharmacy Factory (Shandong, China). PAMAM dendrimers were purchased from Dendritic Nanotechnologies Inc. (MI, USA). All other chemicals used were purchased from MERCK (Germany). All other materials and chemicals were of analytical or reagent grade.

Preparation of biotinylated PAMAM dendrimer. The method used for the preparation of Biotin-conjugated PAMAM G4 $\mathrm{NH}_{2}$ dendrimers was as follows. Briefly, $1.0 \mathrm{ml}$ of PAMAM G4 Dendrimer (i.e. $10 \mathrm{mg}$ in $2 \mathrm{ml}$ of $0.1 \mathrm{M}$ phosphate buffer, $\mathrm{pH} 9.0$ ) was added to sulfoNHS-LC- Biotin (1:30 molar ratio) and stirred for $2 \mathrm{~h}$ at room temperature. The resulting mixture was then dialyzed (1000 Da molecular weight cut off) for $24 \mathrm{~h}$ using 10\% MES buffer (to prevent bursting of the dialysis bag) in deionized water to remove the unconjugated fraction of biotin. This mixture was finally lyophilized to obtain powder (Mamede et al., 2003).

Experimental design. The statistical experimental design was applied to optimize the concentration of biotinylated PAMAM and PTX using $3^{2}$ full factorial designs (Marek et al., 1997). The selected independent variables were the concentration of PTX (A, mg) and the concentration of biotinylated PAMAM dendrimer (B, mg) which were applied at three levels $(-1,0,+1)$. The Drug Loading (DL) $\left(\mathrm{Y}_{1} \%\right)$ and Drug Release (DR) $\left(\mathrm{Y}_{2} \%\right)$ were selected as the dependent factors. The statistical experimental data were analyzed using the DesignExpert $^{\mathbb{R}}$ Software. Table 1 shows the experimental design with 2 independent variables (A, B) at 3 different levels $(-1,0,+1)$. The contour plots were constructed to study the effects of the independent variables on the dependent variables.

Preparation of biotinylated dendrimer-PTX complexes. he batches were prepared by dissolving the required amount of biotinylated PAMAM G4 dendrimer with $-\mathrm{NH}_{2}$ surface groups plus their unconjugated native counterparts in up to $5 \mathrm{ml}$ distilled water and by dissolving PTX in approximately $10-12 \mathrm{ml}$ of deionized water. After complete dissolution of the drug, the dendrimer solution was added drop-wise to the drug solution with continuous stirring. The whole reaction was carried out in round-bottomed flask (RBF) at room temperature and left for completion of reaction for 2 days. Then, it was dialyzed overnight. The resulting complex was lyophilized to obtain a white product. Subsequently, the prepared drug-dendrimer complex was evaluated for drug loading efficiency, encapsulation efficiency, and for its size and stability (Yellepeddi et al., 2013; Yellepeddi et al., 2009).

\section{Evaluation of biotinylated PAMAM dendrimer}

Hydroxyazobenzene-2-carboxylic acid (HABA) assay. HABA assay is one of the chemical methods to assess the degree of biotinylation of the dendrimer. The Avidin/HABA reagent was prepared. For this, $600 \mu \mathrm{l}$ of HABA (i.e. $24.4 \mathrm{mg}$ in $9.9 \mathrm{~mL}$ of water and $0.1 \mathrm{~mL}$ of 1 $\mathrm{N} \mathrm{NaOH}$ ) solution was added with $12 \mathrm{mg}$ of avidin to 20 $\mathrm{mL}$ of phosphate buffered saline ( $\mathrm{pH}$ 7.4). Then, $100 \mu \mathrm{l}$ of biotinylated dendrimer was dissolved in deionized water followed by addition of $900 \mu \mathrm{L}$ of avidin-HABA solution and the absorbance of the mixture was measured.

\section{Evaluation of biotinylated dendrimer-PTX complex}

Drug loading efficiency of PTX-loaded dendrimer. The quantitative detection of PTX was performed using HPLC method. The system consisted of Beckman Coulter HPLC with $250 \times 4.6 \mathrm{~mm}$ i.d. column. The combination of water and acetonitrile at the ratio of 70:30 was

Table 1. Variables and the applied three levels.

\begin{tabular}{lll}
\hline Independent Variable & Low level $(-1)$ & Medium level $(0)$ \\
\hline $\mathrm{A}=$ Drug conc. $(\mathrm{mg})$ & 10 & 15 \\
$\mathrm{~B}=$ Biotinylated PAMAM conc. $(\mathrm{mg})$ & 10 & 20 \\
\hdashline Dependent Variables & & 30
\end{tabular}

$\mathrm{Y} 1=\%$ Encapsulation efficiency 
Table 2. \% Drug Loading (Y1) and Drug Release (Y2)

\begin{tabular}{|c|c|c|c|c|}
\hline Batch No. & Drug conc. (mg) & Bio-PAMAM conc. (\%) & \% Drug Loading & $\%$ Drug Release \\
\hline F1 & 15 & 20 & 8.69 & 87.96 \\
\hline $\mathrm{F} 2$ & 20 & 20 & 10.98 & 79.67 \\
\hline F3 & 15 & 20 & 7.43 & 86.75 \\
\hline $\mathrm{F} 4$ & 10 & 10 & 3.21 & 99.45 \\
\hline F5 & 15 & 30 & 11.89 & 72.87 \\
\hline F6 & 15 & 20 & 9.12 & 85.29 \\
\hline F7 & 10 & 30 & 11.01 & 91.97 \\
\hline F8 & 10 & 20 & 5.99 & 98.65 \\
\hline F9 & 20 & 30 & 12.09 & 70.12 \\
\hline F10 & 15 & 20 & 7.76 & 86.48 \\
\hline F11 & 15 & 10 & 4.54 & 97.64 \\
\hline F12 & 20 & 10 & 5.07 & 94.45 \\
\hline F13 & 15 & 20 & 9.87 & 86.65 \\
\hline
\end{tabular}

used with optimum flow rate and the sample volume of $5 \mu \mathrm{l}$ and the detection was done at the wavelength of $227 \mathrm{~nm}$, at room temperature.

In vitro drug release of PTX from biotinylated dendrimer-PTX complex. Briefly, $10 \mathrm{mg}$ of the dendrimer-PTX complex was dissolved in $10 \mathrm{ml}$ of phosphate buffered saline (PBS) $\mathrm{pH}$ 7.4. The solution was placed in dialysis bag which was kept in $100 \mathrm{~mL}$ of the buffered solution ( $\mathrm{pH}$ 7.4). The solutions were then incubated in an incubation shaker at room temperature at $200 \mathrm{rpm}$. At regular intervals, the samples from the buffer solution were removed for measurement. In vitro release pattern was recorded for $72 \mathrm{~h}$.

Differential Scanning Calorimetry. To assess the integrity of PTX-dendrimer complex the thermograms of the physical mixture of the dendrimer and PTX were compared to the dendrimer-PTX complex using PerkinElmer DSC7, USA. The pre-weighed sample (up to $5 \mathrm{mg}$ ) was kept in a hermetically sealed pan and placed into the sample cell of the instrument equipped with a cooling system. The temperature was set at $-100^{\circ} \mathrm{C}$ with a constant increase of $5^{\circ} \mathrm{C} / \mathrm{min}$. The instrument was purged with high purity gas nitrogen at a flow rate of 50 $\mathrm{mL} / \mathrm{min}$ (Yellepeddi et al., 2009).

Cellular uptake study. To perform the study, the OVCAR-3 or HEK-293T cells were seeded in 12-well plate 2 days before the experiment. The cells were incubated with $1 \mathrm{mM}$ biotin for $30 \mathrm{~min}$ and incubated at room temperature for $1 \mathrm{~h}$ followed by 2-3 times washing with PBS $\mathrm{pH}$ 7.4. Then the cells were suspended in $100 \mu \mathrm{L}$ PBS with 1\% fetal bovine serum. The intensity was measured using fluorescence-activated cell sorting.

\section{RESULTS}

\section{Preparation of biotinylated PAMAM dendrimer}

The high yield of PAMAM dendrimer (85-87\%) was obtained during its biotinylation. The white fibrous solid was obtained as the final product (Majoros et al., 2006). The extent of biotinylation of PAMAM dendrimer was quantified using HABA assay. The biotinylation reagent used for the reaction i.e. sulfo-NHS-LC biotin has 6-aminocaproic acid spacer, which increases the distance between the dendrimer and biotin. It also facilitates the encapsulation of the drug and ligand attachment by reducing the steric hindrance of bulky biotin ring on the PAMAM dendrimer (Kojima et al., 2000).

\section{Preparation of biotinylated dendrimer-PTX complexes}

The reaction between the dendrimers and PTX was carried out using different reaction media like sodium chloride $(0.9 \%)$, water etc., to achieve the maximal encapsulation of the drug. With water as the medium for dendrimers and PTX reaction the encapsulation efficiency was higher $(10-12 \%)$ than in case of sodium chloride $(0.1-3 \%)$. The reason for this may be the requirement of rapid hydrolysis for high ligand exchange. The biotinylated PAMAM- PTX complex had the size of $8.45 \pm 1.28 \mathrm{~nm}$ (Wilbur et al., 1998). The stability of dendrimer-PTX complex was assessed in 100\% PBS. The results obtained, suggested that all dendrimer-PTX complexes were stable and showed no aggregation when kept in serum for up to 7 days at room temperature (Kirkpatrick et al., 2011).

\section{Evaluation of the dendrimer-drug complex}

Statistical analysis of \% Drug Loading (DL) and \% Drug Release (DR)

The encapsulated drug content in PTX-dendrimer ranged from $12.09 \%$ to $3.21 \%$. The percentage of drug loading of PTX loaded biotinylated dendrimer is presented in Table 2. The in vitro release of PTX from the complex was determined in $\mathrm{pH} 7.4$ phosphate buffer saline. The samples from each time point were withdrawn and PTX concentration was estimated. The optimized batch (Run 9) showed up to $70.12 \%$ release in $72 \mathrm{~h}$ (Fig. 1) (Reedijk and Lohman, 1985). The reason for the higher release may be the presence of chloride ions in PBS. This fact can be utilized for targeting as the tumor sites have acidic $\mathrm{pH}$. The statistical data is shown in Table 2 (Nishiyama \& Kataoka, 2001; Nishiyama et al., 2001). $3^{2}$ full-factorial experimental designs were used to evaluate the effect of the independent variables on the dependent variables. The PTX conc. (A) and Biotinylated PAMAM conc. (B) were selected as independent variables while \% Drug Loading (Y1) and \% Drug Release (Y2) were selected as the dependent variables during the formulation. The results obtained are shown in Table 2. These values were compared with observed and predict- 


\section{PTX release from dendrimer complex}

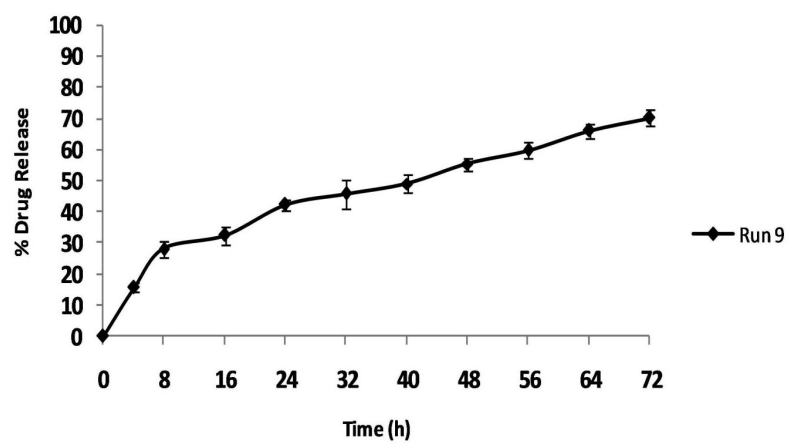

Figure 1. In vitro drug release of PTX from the optimized formulation.

ed values and the residuals for all the batches are shown in Table 3. The model equations obtained for DL (Y1) and DR (Y2) were as follows:

$\mathrm{DL}=+8.28+1.32 \mathrm{~A}+3.70 \mathrm{~B} \quad(1)$

$\mathrm{DR}=+68.75+5.40 \mathrm{~A}+13.22 \mathrm{~B}$

A positive value in the above regression equation represents the synergistic effect of the independent variable while a negative value represents the antagonistic effect.

There is only a $0.01 \%$ chance that a Model F-Value obtained is due to some error and not a result of the experimentation. Values of "Prob> F" less than 0.0500 indicated that the model terms (independent variables) are significant i.e. they had some characteristic effect on the dependent variables. In this case $\mathrm{A}, \mathrm{B}$ and $\mathrm{AB}$ were significant model terms. Values greater than 0.1000 indicate that the model terms were not significant. The effects of $A$ and $B$ and of their interaction on the DL and DR are shown in the contour plot.

DSC

PTX exhibited characteristic peak representing an endothermic transition at $223^{\circ} \mathrm{C}$. Blank PAMAM G4 $\mathrm{NH}_{2}$ showed an endothermic peak at $139^{\circ} \mathrm{C}$. These both peaks could be observed in Biotinylated PAMAM PTX physi-

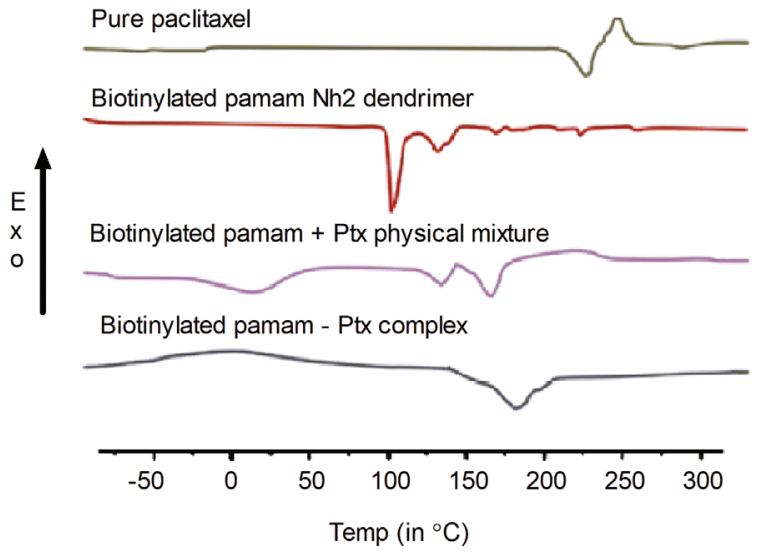

Figure 2. DSC thermograph of Pure Drug, Biotinylated PAMAM dendrimer, Physical mixture of Pure drug and Biotinylated dendrimer, Biotinylated PAMAM-PTX complex.

cal mixture, while being absent in biotinylated PAMAM G4 $\mathrm{NH}_{2}$-PTX complex which proves the complete complex formation between dendrimer and the drug as shown in Fig. 2.

In vitro cellular uptake

The FITC labeling showed the intensity of cellular uptake by OVCAR-3 cells and HEK 293T cells, Fig. 3. It can be clearly observed that the intensity of fluorescence in OVCAR-3 cells was higher than in HEK 293T

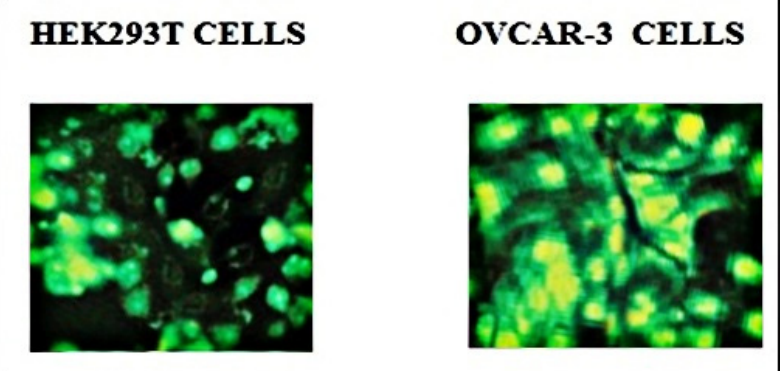

Figure 3. In vitro cellular uptake of PTX by HEK293T (normal) cells and OVCAR-3 (cancerous) cells

Table 3. Statistics of response of variable $\mathrm{Y} 1$ and $\mathrm{Y} 2$.

\begin{tabular}{|c|c|c|c|c|c|c|}
\hline \multirow{2}{*}{ Batch No. } & \multicolumn{3}{|c|}{ \% Drug Release } & \multicolumn{3}{|c|}{$\%$ Drug Loading } \\
\hline & Actual & Predicted & Residual & Actual & Predicted & Residual \\
\hline $\mathrm{F} 1$ & 69.45 & 68.75 & 0.70 & 74.56 & 74.53 & -0.40 \\
\hline $\mathrm{F} 2$ & 75.00 & 74.16 & 0.84 & 80.45 & 82.22 & -1.77 \\
\hline F3 & 68.24 & 68.75 & -0.51 & 72.34 & 74.53 & -2.19 \\
\hline $\mathrm{F} 4$ & 49.34 & 50.12 & -0.78 & 45.00 & 44.46 & 0.54 \\
\hline F5 & 82.23 & 81.98 & 0.25 & 95.00 & 93.81 & 1.19 \\
\hline F6 & 70.23 & 68.75 & 1.48 & 75.23 & 74.53 & 0.70 \\
\hline F7 & 76.12 & 76.57 & -0.45 & 87.23 & 89.20 & -1.97 \\
\hline F8 & 64.23 & 63.35 & 0.88 & 70.15 & 66.83 & 3.32 \\
\hline F9 & 85.12 & 87.38 & -2.26 & 99.00 & 98.42 & 0.58 \\
\hline F10 & 68.27 & 68.75 & -0.48 & 75.53 & 74.53 & 0.70 \\
\hline F11 & 52.78 & 55.55 & -2.75 & 51.40 & 55.24 & 3.84 \\
\hline F12 & 62.00 & 60.93 & 1.07 & 69.12 & 66.03 & 3.09 \\
\hline F13 & 70.78 & 68.75 & 2.03 & 74.13 & 74.53 & -0.40 \\
\hline
\end{tabular}


Table 4. Summary of regression analysis results for variable $\mathrm{Y} 1$ and $\mathrm{Y} 2$

\begin{tabular}{|c|c|c|c|c|c|c|}
\hline Models & $\mathrm{R}^{2}$ & Adjusted $\mathrm{R}^{2}$ & Predicted $\mathrm{R}^{2}$ & Std. Dev & Press & Remarks \\
\hline \multicolumn{7}{|l|}{ VariableY1 } \\
\hline Linear & 0.9065 & 0.8878 & 0.8361 & 0.98 & 16.71 & suggested \\
\hline $2 \mathrm{FI}$ & 0.9080 & 0.8774 & 0.7190 & 1.02 & 28.64 & - \\
\hline Quadratic & 0.9201 & 0.8630 & 0.5269 & 1.08 & 48.22 & - \\
\hline Cubic & 0.9606 & 0.9054 & 0.8812 & 0.90 & 12.11 & - \\
\hline \multicolumn{7}{|l|}{ Variable Y2 } \\
\hline Linear & 0.9812 & 0.9775 & 0.9641 & 1.53 & 44.79 & suggested \\
\hline $2 \mathrm{FI}$ & 0.9839 & 09785 & 0.9545 & 1.49 & 56.75 & - \\
\hline Quadratic & 0.9903 & 0.9834 & 0.9382 & 1.31 & 77.18 & - \\
\hline Cubic & 0.9977 & 0.9897 & 0.9826 & 1.03 & 21.69 & - \\
\hline
\end{tabular}

Table 5. ANOVA MODEL for $\mathrm{Y} 1$ and $\mathrm{Y} 2$

\begin{tabular}{|c|c|c|c|c|c|}
\hline Source & DF & Sum of squares & Mean square & F value & $P$ value \\
\hline Model for Y1 & 2 & 92.40 & 46.20 & 48.49 & $<0.0001$ \\
\hline A & 1 & 10.48 & 10.48 & 11.00 & $<0.0078$ \\
\hline B & 1 & 81.92 & 81.92 & 85.98 & $<0.0001$ \\
\hline Model for Y2 & 2 & 1224.69 & 612.34 & 261.10 & $<0.0001$ \\
\hline$A$ & 1 & 175.28 & 175.28 & 74.74 & $<0.0001$ \\
\hline B & 1 & 1049.40 & 1049.40 & 447.46 & $<0.0001$ \\
\hline
\end{tabular}

cells. This experiment proved that the cancerous cells are characterized by higher uptake of the biotinylated dendrimer-drug complexes.

\section{DISCUSSION}

The biotinylation strategy is an attractive way used in many novel delivery systems in order to enhance the targeting potential of the system (Tripodo et al., 2014). It was proved as a very useful alternative for drugs like $\mathrm{Pa}-$ clitaxel which are poorly soluble in water and exert some cytotoxic effects (Ma, 2013). One of the methods to overcome the systemic toxicity of the PTX is the formation of polymer-drug conjugates. The formation of the polymer complex requires the availability of a suitable ligand. The PAMAMG4 dendrimer has 64 terminal amine groups and it was assumed that this might contribute to the increased uptake potential (Yellepeddi et al., 2009).

The method used, i.e. biotinylation was rapid with the product yield $>80-85 \%$. The reaction involved the use of sulfo-NHS-LC-biotin reagent which is water soluble by nature. Biotin is a small molecule and when conjugated to the dendrimer causes enhanced tumor permeation. The availability of the spacer between the covalently bound molecule and the bicyclic ring due to the presence of 6-aminocaproic is the ultimate advantage of using this reagent. The greater separation distance is not only the reason for a better binding potential for biotin receptors expressed on the cancer cells but also reduces the steric hindrance of the biotin ring.
The biotinylated dendrimer showed increased uptake which may be the result of a large cationic surface charge density which allowed an increased interaction with the receptors on the cell membrane. We showed that the biotinylated dendrimer uptake was higher in OVCAR-3 than in HEK293T cells. This difference might be the result of the fact that tumor cells grow rapidly and thus require more biotin for the expression of oncogenes and cell proliferation.

From the analysis of the values in Table 2, it can be stated that Paclitaxel and Biotinylated PAMAM concentration had a significant effect on DL and DR. Table 3 showed a high correlation between the actual and predicted values. This correlation was further confirmed through statistical analysis as shown in Table 4. The value of correlation coefficient (R2) showed a good fit. The results in Table 5 showed that DL and DR were affected by the independent variables $\mathrm{A}$ and $\mathrm{B}$. The interaction variable $A B$ showed how the $D L$ changed when the 2 variables were simultaneously changed. As shown in Table 5, the Model F-value of 1586.38 and 261.10 (for DL and DR respectively) implied that the model is significant. Cellular accumulation of the PTX was measured by HPLC.

The effects of $\mathrm{A}$ and $\mathrm{B}$ and their interaction on DR and DL are shown in Fig. 4 and in Fig. 5 as contour plots. In case of Biotinylated PAMAM, as the concentration of dendrimer increased, the DL, as well as the Drug Release, also increased. The increase in drug concentration simultaneously causes increase in drug loading and release (Choi et al., 2004). 


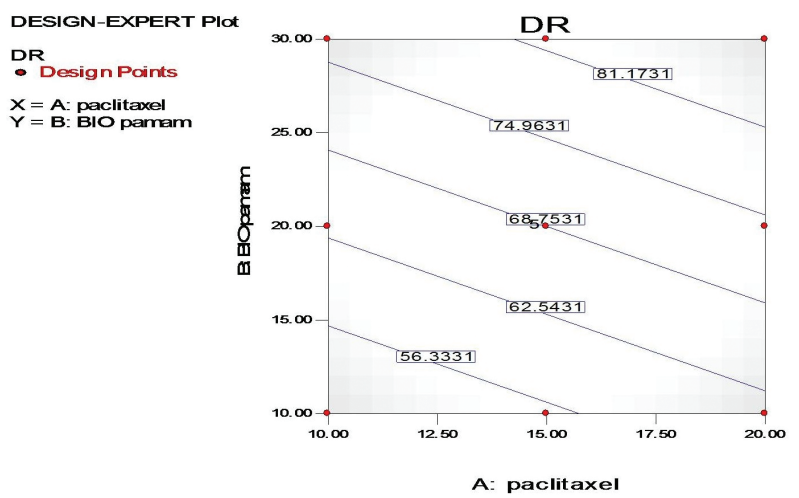

Figure 4. The effect of individual variables on Drug Release \% presented as contour plot.

\section{CONCLUSION}

Biotinylated PAMAM dendrimers containing amine as a surface group were prepared as carriers for PTX to enhance the therapeutic effect with a subsequent reduction in its toxicity. PTX was successfully encapsulated by biotinylated dendrimers with amine surface functionality. From the various experiments, it was observed that the dendrimer-PTX complex was more effective than free PTX in the ovarian cancer. The cellular uptake data revealed the successful accumulation of the complex in cancerous cells demonstrating its higher uptake resulting in increased bioavailability.

The overall results confirmed the fact that biotinylated PAMAM dendrimer has the potential as a drug carrier for targeting the ovarian cancer cells.

\section{Disclosure Statement}

The authors declared no conflict of interest.

\section{REFERENCES}

Bankhead CR, Kehoe ST, Austoker J (2005) Symptoms associated with diagnosis of ovarian cancer: a systematic review. BJOG 112: $857-$ 865. 10.1111/j.1471-0528.2005.00572.x

Choi JS, Nam K, Park JY, Kim J Bin, Lee JK, Park JS (2004) Enhanced transfection efficiency of PAMAM dendrimer by surface modification with l-arginine. J Control Release 99: 445-456. 0.1016/j. jconrel.2004.07.027

Cragun JM (2011) Screening for ovarian cancer. Cancer Control 18: 1621

Ferris JS, Daly MB, Buys SS, Genkinger JM, Liao Y, Terry MB (2014) Oral contraceptive and reproductive risk factors for ovarian cancer within sisters in the breast cancer family registry. Brit J Cancer 110: 1074-1080. 10.1038/bjc.2013.803

Geggel L, Writer S (2014) Ovarian Cancer: Symptoms and Treatment. Live Science 08: 26

Harley I, Rosen B, Risch HA, et al (2008) Ovarian cancer risk is associated with a common variant in the promoter sequence of the mismatch repair gene MLH1. Gynecologic Oncol 109: 384-387. 10.1016/j. ygyno.2007.11.046.

Kesharwani P, Iyer AK (2015) Recent advances in dendrimer-based nanovectors for tumor-targeted drug and gene delivery. Drug Discovery Today 20: 536-547. doi:10.1016/j.drudis.2014.12.012

Kirkpatrick GJ, Plumb JA, Sutcliffe OB, Flint DJ, Wheate NJ (2011) Evaluation of anionic haf generation poly(amidiamine) dendrimers as delivery vehicle for active component of anticancer drug cisplatin. I Inorg Biochem 105: 1115-1122. 10.1016/j.jinorgbio.2011.05.017

Kojima C, Kono K, Maruyama K, Takagishi T (2000) Synthesis of polyamidoamine dendrimers having poly(ethylene glycol) grafts and their ability to encapsulate anticancer drugs. Bioconjug Chem 11: 910917. $10.1021 / \mathrm{bc} 0000583$

Lifford KJ, Fraser L, Rosenthal AN, Rogers MT, Lancastle D, Phelps C, Watson EK, Clements A, Iredale R, Jacobs I, Menon U, Brain KE (2012) Withdrawal from familial ovarian cancer screening for

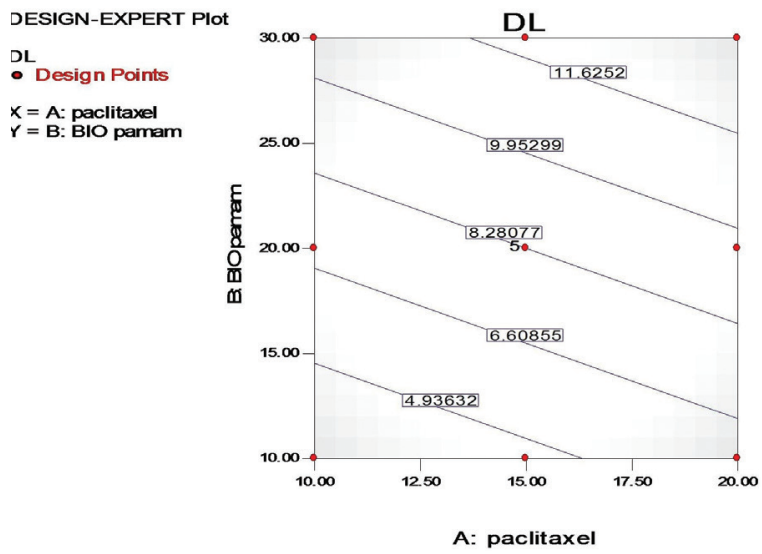

Figure 5. The effect of individual variables on Drug Loading \% presented as contour plot.

surgery: Findings from a psychological evaluation study (PsyFOCS). Gynecologic Oncol 124: 158-163. 10.1016/i.ygyno.2011.09.015

Ma P, Mumper RJ (2013) Paclitaxel Nano-Delivery Systems: A Comprehensive Review. I Nanomedicin Nanotechnol 4: 1000164. 10.4172/2157-7439.1000164

Majoros IJ, Myc A, Thomas T, Mehta CB and Baker JR Jr (2006) PAMAM dendrimer-based multifunctional conjugate for cancer therapy: synthesis, characterization, and functionality. Biomacromolecules 7: 572-579. 10.1021/bm0506142

Mamede M, Saga T, Kobayashi H, Ishimori T et al (2003) Radiolabelling of avidin with very high specific activity for internal radiation therapy of intraperitoneally disseminated tumors. Clin Cancer Res 9: 3756-3762

Marek M, Kaiser K, Gruber HJ (1997) Biotinepyrene conjugates with poly(ethylene glycol) spacers are convenient fluorescent probes foe avidin and steptavidin. Bioconjug Chem 8: 560-566. 10.1021/ bc970088e

Markman M, Mekhail TM (2002) Paclitaxel in cancer therapy. Expert Opin Pharmacotherapy 3: 755-766. http://dx.doi. org/10.1517/14656566.3.6.755

McDermott M, Eustace AJ, Busschots S, et al (2014) In vitro Development of chemotherapy and targeted therapy drug-resistant cancer cell lines: a practical guide with case studies. Frontiers Oncol 4: 40. 10.3389 / fonc. 2014.00040

Moley KH, Colditz GA (2016) Effects of obesity on hormonally driven cancer in women. Sci Transl Med 8: 323ps3. 10.1126/scitranslmed.aad8842

Nagel CI, Backes FJ, Hade EM, Cohn DE, Eisenhauer EL, O'Malley DM, Fowler JM, Copeland LJ, Salani R (2012) Effect of chemotherapy delays and dose reductions on progression free and overall survival in the treatment of epithelial ovarian cancer. Gynecologic Oncol 124: 221-224. 10.1016/j.ygyno.2011.10.003

Narvekar M, Xue HY, Eoh JY, Wong HL (2014) Nanocarrier for poorly water-soluble anticancer drugs - barriers of translation and solutions. AAPS Pharm Sci Tech 15: 822-833. 10.1208/s12249-0140107-x

Nishiyama N, Kataoka K (2001) Preparation and characterization of size-controlled polymeric micelle containing cisdichlorodiammineplatinum(II) in the core. J Control Release 74: 8394. https://doi.org/10.1016/S0168-3659(01)00314-5

Nishiyama N, Kato Y, Sugiyama Y, Kataoka K (2001) Cisplatin loaded polymer metal complex micelle with time modulated decaying property as novel drug delivery system. Pharm Res 18: 1035-1104. https://doi.org/10.1023/A:101090891

Non (1993) Paclitaxel (taxol) for ovarian cancer. Med Let Drugs Ther 35: 39-40. 8097551

Petrucelli N, Daly MB, Pal T (2016) BRCA1- and BRCA2-Associated hereditary breast and ovarian cancer. Gene Reviews ${ }^{(\text {B }}$ (Internet). Seattle (WA): University of Washington, Seattle; 1993-2017. Available from: https://www.ncbi.nlm.nih.gov/books/NBK1247/

Rao SV, Anderson KW, Bachas LG (1997) Determination of extent of protein biotinylation by fluorescence binding assay. Bioconjug Chem 8: $94-98.10 .1021 /$ bc960080p.

Razi S, Ghoncheh M, Mohammadian-Hafshejani A, Aziznejhad H, Mohammadian M, Salehiniya H (2016) The incidence and mortality of ovarian cancer and their relationship with the Human Development Index in Asia. E Cancer Med Sci 10: 628. 10.3332/ecancer.2016.628

Reedijk J, Lohman PH (1985) Cisplatin: synthesis, antitumor activity and mechanism of action. Pharn Weekbl Sci 7: 173-180. https://doi. org/10.1007/BF02307573 
Rosen B, Laframboise S, Ferguson S, Dodge J, Bernardini M, Murphy J, Segev Y, Sun P, Narod SA (2014) The impacts of neoadjuvant chemotherapy and of debulking surgery on survival from advanced ovarian cancer. Gynecologic Oncol 134: 462-467. 10.1016/j. ygyno.2014.07.004

Russel Jones G, Mc Tavish K, Mc Ewan J (2004) Vitamin targeting as a potential mechanism to increase drug uptake by tumors. I Inorg Biochem 98: 1625-1633. doi: 10.1016/j.jinorgbio.2004.07.009

Takechi R, Taniguchi A, Ebara S, Fukui T, Watanabe T (2008) Biotin deficiency affects the proliferation of human embryonic palatal mesenchymal cells in culture. I Nutrit 138: 680-684. http://www.ncbi. nlm.nih.gov/pubmed/18356320

Tripodo G, Mandracchia D, Collina S, Rui M, Rossi D (2014) New perspectives in cancer therapy: the biotin-antitumor molecule conjugates. Med Chem S1: 004. 10.4172/2161-0444.S1-004

Wilbur DS, Pathare PM, Hamlin Dk, Buhler KR, Vessella RL (1998) Biotin reagents for antibody pretargeting. 3. Synthesis, radioiodination, and evaluation of biotinylated starburst dendrimers. Bioconjug Chem 9: 813-825. 10.1021/bc980055e

Wu Q, Yang Z, Nie Y, Shi Y, Fan D (2014) Multi-drug resistance in cancer chemotherapeutics: Mechanisms and lab approaches. Cancer Lett 347: 159-166. 10.1016/j.canlet.2014.03.013
Yang W, Cheng Y, Xu T, Wang X, Wen LP (2009) Targeting cancer cells with biotin dendrimer conjugates. Eur J Med Chem 44: 862868. 10.1016/j.ejmech.2008.04.021

Yap TA, Carden CP, Kaye SB (2009) Beyond chemotherapy: targeted therapies in ovarian cancer. Nat Rev Cancer 9: 167-181. 10.1038/ nrc2583

Yellepeddi VK, Kumar A, Palakurthi S (2009) Surface modified poly(amino)amine dendrimers as diverse nanomolecules for biomedical applications. Expert Opinion Drug Delivery 6: 835-850. $10.1517 / 17425240903061251$

Yellepeddi VK, Kumar A, Palakurthi S (2009) Biotinylated poly(amido) amine (PAMAM) dendrimers as carriers for drug delivery to ovarian cancer cells in vitro. Anticancer Res 29: 2933-2943

Yellepeddi VK, Vangara KK, Palakurthi S (2013) Poly (amido) amine (PAMAM) dendrimer-cisplatin complexes for chemotherapy of cisplatin-resistant ovarian cancer cells. J Nanopart Res 15: 1874. 10.1007/s11051-013-1874-0

Zarchi MK, Rouhi M, Abdolahi AH, Hekmatimoghaddam S (2013) The effect of assisted reproductive technologies on gynecological cancer: report of our experiences and literature review. Int J Biomedic Sci: IJBS 9: 129-134 